\title{
Tumor microenvironment characterization and Immune infiltration in head and neck squamous cell carcinoma
}

\section{Zenghong Wu}

Huazhong University of Science and Technology Tongji Medical College

\section{Xun Niu}

Huazhong University of Science and Technology

\section{Xi-Yue Xiao}

Huazhong University of Science and Technology

Xiong Chen ( $\nabla$ cqij74@126.com )

Zhongnan Hospital of Wuhan University https://orcid.org/0000-0003-4977-6478

\section{Research article}

Keywords: Immune infiltration, Tumor microenvironment, tumor mutational burden, immunotherapy, HNSCC

Posted Date: February 5th, 2020

DOl: https://doi.org/10.21203/rs.2.19457/v2

License: (c) (i) This work is licensed under a Creative Commons Attribution 4.0 International License. Read Full License 


\section{Abstract}

Background : Head and neck squamous cell carcinoma (HNSCC), a basic malignant tumor of the head and neck distinct. As a famous heterogeneous disease, the jobs of immune cells inside the tumor-related as yet missing for HNSCC, particularly in current immunotherapy.

Method: We explored the TME, TMB and evaluate the 22 TIICs subsets of immune response based on GEO and TCGA database of HNSCC to explore its relationship with atomic subpopulation, survival, function and expression difference and reveal potential targets and biomarkers for immunotherapy.

Results: Observing the download of GSE6631 database contained 22 HNSCC samples and 22 normal samples and TCGA database contained $111 \mathrm{HNSCC}$ and 12 normal tissues. The results suggested that the expression of macrophages $\mathrm{M} 0$ and T cells CD4 memory resting was significant difference and may plays an important role in regulate cancer progression $(P<0.05)$. The result of tumor mutational burden revealed that the most common somatic mutations variant classification was missense mutation, the most common DNA sequence polymorphism type was SNP, the most common single nucleotide variants (SNV) class was $\mathrm{C}>\mathrm{T}$, the variants per sample median was 78 in HNSCC patients. Top 10 mutated genes that related to TMB was TP53, TTN, FAT1, MUC16, CDKN2A, CSMD3, SYNE1, LRP1B, NOTCH1 and PIK3CA. We portrayed the immune scene in detail, uncovering the awesome immune infiltration styles of various subtypes in HNSCC.

Conclusion: The intricate connection between TIIC, TMB and genomic alterations was additionally set up. Our paintings advance the information of immune response and offers significant assets for research to enhance immunotherapy.

\section{Background}

Head and neck squamous cell carcinoma (HNSCC), a typical harmful malignant tumor of the head and neck distinct, which arises from lip, oral cavity, paranasal sinuses, oropharynx, larynx, nasopharynx and other pharynx carcinomas. ${ }^{1}$ As the 6 th normal sort of malignant tumor with an incidence of over 650000 new cases and a 90000 deaths per year worldwide. ${ }^{2}$ Currently, cigarette smoking, alcohol consumption as well as human papilloma virus (HPV) infection are deemed to be risk factors for the occurrence and prognosis of HNSCC. ${ }^{3}$ Unfortunately, because of lack of symptoms in the early stage when detected of HNSCC is usually made at advanced stages and the 5-year survival rate is still under $50 \%$ now, while due to local recurrence and metastasis, which reduces survival rate to $35 \% .{ }^{4}$ The occurrences and progression of HNSCC is a complicated process involving multiple molecules. While, the malignant phenotypes of cancers are characterized by the intrinsic activities of tumor cells as well as by the immune cells recruited to and activated ${ }^{5}$. As a heterogeneous disease, the jobs of immune cells in the tumor-related as yet missing for HNSCC, particularly in current immunotherapy. 
The tumor microenvironment (TME) comprises not only cell types (immune cells, fibroblasts and endothelial cells etc.) but also extracellular parts (hormones, cytokines, extracellular matrix, growth factors, etc.) that are encompassing tumor cells and fed by a vascular network ${ }^{6}$. The TME plays an indispensable role across tumor initiation, progression and even metastasis and effects therapeutic efficacy ${ }^{7}$.Tumor-infiltrating immune cells (TIIC) have been accounted for to be viably targeted by drugs and to connect with the clinical result by structure an ecosystem in the tumor microenvironment to manager cancer progression and have appeared potential prognostic value ${ }^{8}$. There is a complicated interaction which considerable prognostic relevance as the immune system has a double job by means of assisting both host barrier and tumor progression ${ }^{9}$. Upregulation of inhibitory immune checkpoints with the aid of TIICs by tumor cells prompts tumor avoidance from host immunosurveillance ${ }^{10}$. Chromatin modifiers lead to CD8+ T-cell prohibition and antigen presentation limit of HNSCC ${ }^{11}$. Moreover, no past investigation has revealed insight into the prognostic estimation of these TIICs subpopulation. Past examinations assessed the estimation of these TIIC by flow cytometry and immunohistochemistry $(\mathrm{IHC})^{12}$ and are limited by the quantity of fluorescent channels accessible as well as few immune cell types can be assessed immediately ${ }^{13}$. A metagene instrument CIBERSORT utilizes deconvolution of mass gene expression information and a sophisticated algorithm for quantification the cellular element of immune reaction and cell kinds in heterogeneous samples has significantly extended the capability of the genomic database ${ }^{14-15}$.Tumor mutational burden (TMB) has been depicted as indicator of immunological reaction and tumor behavior ${ }^{16-17}$, mutation formation advances carcinogenesis by means of activation or inactivation of genes and related pathways, in this way creating novel peptide sequences which can animate immune reaction ${ }^{18}$. High TMB may additionally constitute a high underlying wide variety of drivers and imply a higher-risk tumor. As is a developing biomarker of affectability to immune checkpoint inhibitors and has been appeared to be more significantly connected with reaction to PD-1 and PD-L1 blockade immunotherapy than PD-1 or PD-L1 expression ${ }^{19}$. In this study, we carried out CIBERSORT to explored the TME, TMB and quantify the 22 TIICs subsets of immune reaction based on GEO and TCGA database of HNSCC to explore its association with molecular subpopulation, survival, function and expression difference and reveal potential targets and biomarkers for immunotherapy.

\section{Materials And Methods}

\section{Data acquisition.}

Freely accessible datasets with gene expression profiles and corresponding visualization data were identified and downloaded. The crude information from the microarray data collections produced by Affymetrix were downloaded from the Gene-Expression Omnibus and using the RMA algorithm for background adjustment. Information on gene expression, somatic mutations, copy number and corresponding clinical information were recognized and downloaded from level 4 gene-expression data (FPKM normalized) of The Cancer Genome Atlas HNSCC cohort. Due to the histological types and multiple anatomical sites and of HNSCC so we mainly download larynx data from TCGA. For TCGA data set, RNA-sequencing data (FPKM values) were changed into transcripts per kilobase million (TPM) 
values, which are progressively like those subsequent from microarrays and easy comparable between samples ${ }^{20}$. The clinicopathological data collected included sex, age, stage, grade, T-stage, M-stage, $\mathrm{N}$ stage, survival status and survival duration in days. Data were examined with the $\mathrm{R}$ (version 3.5.3) and $\mathrm{R}$ Bioconductor packages. We use perl language for immune cell matrix filtering according to $P$ less than 0.5 .

\section{Assessment of immune infiltration.}

Inference of infiltrating cells in the TME and to measure the proportions of immune cells and cell types in the HNSCC heterogeneous samples, a metagene tool CIBERSORT utilizes deconvolution of mass gene expression data and a sophisticated algorithm used and has been confirmed by fluorescence activated cell sorting (FACS). These TIIC comprised macrophages (M0/M1/M2 macrophages), 7 T-cell types resting memory CD4+ T cells, (T follicular helper [Tfh] cells, activated memory CD4+ T cells, $\gamma \delta T$ cells, Tregs, CD8+ T cells and naïve CD4+ T cells), resting natural killer (NK) cells, resting/activated mast cells, activated NK cells, resting dendritic cells (DC), memory B cells, activated DC, monocytes, naïve B cells, plasma cells, eosinophils and neutrophils.

\section{Assessment of tumor mutational burden.}

TMB is the total number of mutations per megabase of tumor tissue. In general terms, the mutation density of a tumor gene, that is, the average number of mutations in the tumor genome, including the total number of gene coding errors, base substitutions, gene insertions, or deletion errors. The larger the $\mathrm{TMB}$, the easier it is to be discovered by immune cells, and the easier it is to become a target for tumor immunity, so that the more likely it is to be effective for immunotherapy. We described the copy number and somatic mutations characteristic based on TCGA databased and then analyzed the connection between TMB and patients' clinical factors, including age, gender, grade and TNM stage and we also analyzed the effect of TMB on survival. TCGA workflow type based on VarScan2 Variant Aggregation and Masking. Differentially expressed genes (DEG) associated with TMB were resolved using the R package

limma ${ }^{21}$, which implements an empirical Bayesian way to estimate gene-expression modifications the use of moderated t tests. DEGs among TMB were controlled by using significance standards as applied in the R package limma. The adjusted $P$ value for multiple testing was calculated the usage of the Benjamini-Hochberg correction ${ }^{22}$.To examine the function of the identified DMGs, biological analyses were performed utilizing GO (cellular components, biological processes and molecular functions) enrichment and KEGG pathway analysis via $R$ package. Finally, we evaluated the related to tumorinfiltrating immune cells and TMB. 


\section{Statistical analysis.}

Statistical analyses have been conducted the use of $\mathrm{R}$ version 3.5.3 and Bioconductor. For examinations of two gatherings, statistical significance for normally distributed variables was evaluated by unpaired Student $t$ tests, and nonnormally distributed variables were revealed by Wilcoxon tests. Each dataset was handled by a weighted average approach to contrast the differences in the composition of TIIC and using boxplot, heatmap, corHeatmap and vioplot to visualization the difference in normal and tumor samples. Overall survival (OS) was characterized as the time interval from the date of diagnosis to the date of death. The listwise deletion technique was utilized to deal with missing data, which excluded the entire sample from the investigation if any single value was absent. Wilcox test analysis was performed to evaluate the differences not only in the gene expression of immune checkpoint molecules but also TMB clinical information between tumor and normal tissues. To recognize differential genes in the GEGs evaluation, we applied the Benjamini-Hochberg way to transform the $P$ values to FDRs. For every single statistical analysis, a $P$-value $<0.05$ was viewed as significant.

\section{Results}

\section{Clinical information.}

Observing the download of GSE6631 database contained 22 HNSCC samples and 22 normal samples and after immune cell matrix filtering according to $P$ value less than 0.5 , the compliance with the inclusion criteria contained $12 \mathrm{HNSCC}$ samples and 10 normal samples. TCGA database contained 111 HNSCC and 12 normal tissues and after immune cell matrix filtering according to $P$ value less than 0.5 , the compliance with the inclusion criteria contained 98 HNSCC and 1 normal tissue, while the clinical patients contained 117 patients and the clinicopathological attributes of these samples are appeared in Table 1.

\section{Distribution of tumor-infiltrating immune cells based on GEO.}

Boxplot, heatmap and corHeatmap visualization showed 22 TIICs subsets of immune response based on GEO (odd number is normal, even number is the tumor) Figure 1A-1C. CorHeatmap can showed interaction between different 22 TIICs and we found the highest co-expression coefficient was between macrophages $\mathrm{M} 1$ and T cells CD4 memory resting, the number was 0.86 indicating that these two immune cells play a synergistic role in HNSCC patients. The lowest co-expression coefficient was between mast cells activated and T cells $C D 8$, the number was -0.71 indicating that these two immune cells play an antagonism role in HNSCC patients. The vioplot showed the different of 22 TIICs between normal and tumor samples Figure 1D. The results suggested that the expression of macrophages $\mathrm{M} 0$ and T cells CD 4 memory resting was significant difference and may plays an important role in regulate cancer progression $(P<0.05)$. 


\section{Distribution of tumor-infiltrating immune cells based on TCGA.}

Boxplot, heatmap and corHeatmap visualization showed 22 TIICs subsets of immune response based on TCGA Figure 2. Boxplot can show 22 TIICs relative percent and heatmap can tell us hierarchical clustering of each immune cells in normal and tumor HNSCC tissues. CorHeatmap can showed interaction between different 22 TIICs and we found the highest co-expression coefficient was between NK cells resting and T cells CD4 memory activated, the number was 0.32 and the lowest co-expression coefficient was between macrophages $\mathrm{M} 0$ and T cells CD8, the number was -0.52 in HNSCC patients.

\section{Tumor TMB variability among cancers.}

The result of tumor mutational burden revealed that the most common somatic mutations variant classification was missense mutation, the most common DNA sequence polymorphism type was SNP, the most common single nucleotide variants (SNV) class was $\mathrm{C}>\mathrm{T}$, the variants per sample median was 78 in HNSCC patients. Top 10 mutated genes that related to TMB was TP53, TTN, FAT1, MUC16, CDKN2A, CSMD3, SYNE1, LRP1B, NOTCH1 and PIK3CA Figure 3A. CorHeatmap showed interaction between different mutated genes and the results showed that there are co-expression relationships among many mutated genes Figure 3B. The gene cloudmap demonstrated that the most remarkable mutated genes were TTN and the waterfall plot tell us that the gene altered in 478 (94.47\%) of 506 samples as well as the relative percent, variant classification of every single mutated genes Figure 3C-3D. There was no statistical difference between TMB and clinical survival time $(P=0.87)$, which may be due to too few samples size and needs more researches to convince. While there was significance different in survival rate between TMB and macrophages M1 $(P=0.009)$ and T cells CD4 naïve ( $P=0.009)$ Figure 4A4B. We then analyzed the relationship between tumor-infiltrating immune cells and patients' clinical factors. Increasing patient age was significantly correlated with mast cells activated fraction $(P=0.049)$; gender was significantly correlated with macrophages $\mathrm{M} 1$ fraction $(P=0.042)$, neutrophils fraction $(P=0.028)$ and T cells gamma delta fraction $(P=0.049)$; grade was significantly related to neutrophils fraction ( $P=0.002)$, T cells CD8 fraction $(P=0.004)$ and macrophages M1 fraction ( $P=0.004)$; T-stage was significantly correlated with $\mathrm{T}$ cells follicular helper fraction $(P=0.037)$ and $\mathrm{N}$-stage was significantly related to T cells regulatory (Tregs) fraction $(P=0.019)$ and dendritic cells activated fraction $(P=0.039)$ Figure 4C-4L.

\section{$T M B$ and differentially expressed genes.}

We then divide TMB into high tumor mutational burden and low tumor mutational burden according to the median value of TMB. To recognize the underlying biological characteristics of TMB, unsupervised 
analysis of 249 DEGs received by the limma package ${ }^{23}$ and the clustering results was showed in Figure 5A. GO enrichment and KEGG pathway analysis of the signature genes was directed using the $\mathrm{R}$ package clusterProfiler. GO analysis results demonstrated that biological processes (BP) of DEGs were significantly enriched in response to acid chemical, connective tissue development and cartilage development et al. Molecular function (MF) were mainly enhanced in extracellular matrix structural constituent. Cell component (CC) of DMGs were primarily enriched in extracellular matrix, presynapse and endoplasmic reticulum lumen et al Figure 5B. KEGG analysis revealed that the DMGs were for the most part enriched in glutathione metabolism. We then examined the connection among TMB and patients' clinical factors and the results revealed that TMB was significantly correlated with patient gender $(P=0.005)$. The vioplot showed the different between 22 TIICs and TMB in TMB subgroups. The results suggested that the expression of $T$ cells regulatory (Tregs) was significant difference and may plays an important role in regulate tumor cells $(P=0.026)$.

\section{Discussion}

In this study, we carried out CIBERSORT to conducted a comprehensive and detailed assessment of the TME, TMB and quantify the 22 TIICs subsets of immune response based on GEO and TCGA database in HNSCC to explore its association with molecular subpopulation, survival, function and expression difference. We also detailed that the constitute of TIIC differs substantially in numerous subtypes and clinical factors of HNSCC. Tumor mutational burden firmly add to the generation of novel tumor epitopes. Knowing whether a more highly-immunogenic tumor consists of a right away link to mutational burden help us understand the mechanistic clarification for observed clinical survival patterns. In our outcomes, TMB was firmly related with TMB among TCGA data, supporting TMB's position as a strong proxy for TME. This ought to be of terrific interest for perspective on the present improvement of immunomodulatory therapies and may be uncover potential biomarkers or targets for immunotherapy or determinants for prognosis.

The significance of immune infiltration for prognosis has been perceived. Macrophages, normally thought of as phagocytic inflammatory cells and play an important role within tumor progression, including facilitating tumor cell invasion and angiogenesis ${ }^{24-26}$. Macrophages are mainly classified into $\mathrm{M0} / \mathrm{M} 1 / \mathrm{M} 2$ type macrophages and $\mathrm{M} 0$ type macrophages can be polarized into M1/M2 type macrophages under specific pathophysiological conditions. Liu et $\mathrm{al}^{27}$ reported that RhoA pathway could obstruction induces extreme elongation (hummingbird phenotype) of M0 macrophages and suggested that $\mathrm{M} 0$ ought to be important considerations in the helpful of bone-marrow-derived macrophages. As subpopulations of $T$ cells, resting memory CD4+ T cells can differentiate to have different capacities (such as separate to CD $8+$ memory T cells to suppress tumor increase) ${ }^{28-29}$. Resting memory CD $4+$ cells are subject to signals from touch with IL-7 and IL-15, yet not MHC class II, for their survival and intermittent homeostatic proliferation ${ }^{30}$. Our results suggested that the expression of macrophages $\mathrm{M} 0$ and T cells CD 4 memory resting was significant difference and may plays an important role in regulate cancer progression. While the other immune cells infiltration was not obvious may be due to the too few 
samples size and needs more researches to conduct. We also analysis the interaction between different 22 TIICs and provided information for future immune cells co-expression study in HNSCC researches.

The result of tumor mutational burden revealed that the most common somatic mutations variant classification was missense mutation, the most common DNA sequence polymorphism type was SNP, the most common single nucleotide variants (SNV) class was $\mathrm{C}>\mathrm{T}$. Changes that modify amino acid sequences are known as missense mutations and these mutations may influence protein stability/structure and damage protein interactions with different biomolecules, translation proteins nonfunctional and advancing tumor progression ${ }^{31}$. For example, BRAF mutation in melanoma and the KRAS G12D mutations in colorectal cancer ${ }^{32-33}$. Identifying potential functional impacts of cancer missense mutations which may help in the location of cancer drivers and illustration of molecular mechanisms in HNSCC. SNP have been discovered for nearly all types of cancers, such as colorectal ${ }^{34}$, breast $^{35}$, prostate ${ }^{36}, \mathrm{HNSCC}^{37}$ and so forth. As a third-generation genetic marker, numerous phenotypic differences in human body, susceptibility to diseases or drugs may be associated with SNP ${ }^{38}$. Top 10 mutated genes that related to TMB was TP53, TTN, FAT1, MUC16, CDKN2A, CSMD3, SYNE1, LRP1B, NOTCH1 and PIK3CA. Kubesova et al ${ }^{39}$ reported that TP53 mutations with low variant allele frequency (VAF) irrespective of disease subtype, driver gene status and cytoreduction during myeloproliferative neoplasm. Mukhopadhyay et al ${ }^{40}$ suggested that ESR2-mutant TP53 combination prognosticates survival in triple negative breast cancer. While in our study we found there was no statistical difference between TMB and clinical survival time $(P=0.87)$ may be due to too few samples size in TCGA database and needs more clinical researches. We then analyzed the relationship between tumor-infiltrating immune cells and patients' clinical factors such as age, gender, grade TNM-stage. Despite the fact that we have uncovered some learning of the clinical factors' utility of mutations, the value of TMB as biomarkers for immunotherapy requires further research.

In order to analysis the relationship between TMB and differentially expressed genes, we divide TMB into high TMB and low TMB according to the median value and explored the functions of this DEGs. Biological processes of DEGs suggested that these genes may play a significant role in tissue development and the response to acid chemical, molecular function were mainly enhanced in extracellular matrix structural constituent and mainly affect cellular structure. KEGG analysis revealed that the DMGs were for the most part enriched in glutathione metabolism. Glutamine (GLN) usually known as a significant metabolite utilized for the development of malignancy cells. GLN consumption restores diminished levels of glutathione on account of chemotherapy and radiotherapy and it encourages recovery processes of the intestine epithelium and immunological system ${ }^{41}$. Our results also suggested that the expression of $T$ cells regulatory (Tregs) was critical distinction between high TMB group and low TMB group indicating Tregs is essential in the tumor immune response. Meanwhile, our study has limitations, one is that our results not verified in clinical samples and cannot provides precise clinical data as well as the relatively few patients, another is that due to the histological types and multiple anatomical sites and of HNSCC the tumor-infiltrating immune cells may vary widely. We will do a lot of related research in the follow-up and eventually translated into the development of new strategies 
for precision cancer medicine. We portrayed the immune scene in detail, uncovering the awesome immune infiltration styles of various subtypes in HNSCC. The intricate connection between TIIC, TMB and genomic alterations was additionally set up. Our paintings advance the information of immune response and offers significant assets for research to enhance immunotherapy.

\section{Abbreviations}

HNSCC: Head and neck squamous cell carcinoma; HPV: human papilloma virus; TME: the tumor microenvironment; TIIC: tumor-infiltrating immune cells; TMB: tumor mutational burden.

\section{Declarations}

\section{Ethics approval and consent to participate}

Not applicable.

\section{Consent to public}

Not applicable.

\section{Availability of data and materials}

Data sharing is not applicable to this article as no datasets were generated or analyzed during the current study.

\section{Competing interests}

The authors declare that they have no competing interests.

\section{Funding}

This work is not supported by grants.

\section{Authors' contributions}


All authors contributed significantly to this work. W.Z.H. designed the research study; W.Z.H. and N.X performed the research study and extracted the data and analyzed the data; W.Z.H., N.X., X.X.Y.; W.Z.H. and C.X. wrote and revised the manuscript. In addition, all authors approved the final draft.

\section{Acknowledgements}

Not applicable

\section{References}

1. Xiao-Nan F, Miao Y, Hua $L$, et al. Comprehensive analysis of competitive endogenous RNAs network associated with head and neck squamous cell carcinoma. Scientific Reports, 2018, 8(1):10544-

2. Torre, L. A., Bray, F., Siegel, R. L., Ferlay, J., Lortet-Tieulent, J., and Jemal, A. Global cancer statistics, 2012. CA Cancer J Clin. 65, 87-108.

3. Magnes T, Egle A, Greil R and Melchardt T: Update on squamous cell carcinoma of the head and neck: ASCO annual meeting. Memo 10: 220-223, 2017.

4. Chin D, Boyle GM, Williams RM, Ferguson K, Pandeya N, Pedley J, Campbell CM, Theile DR, Parsons PG, Coman WB: Novel markers for poor prognosis in head and neck cancer. Int J Cancer 2005, 113:789-797.

5. Ben-Baruch A. Host microenvironment in breast cancer development: inflammatory cells, cytokines and chemokines in breast cancer progression: reciprocal tumor-microenvironment interactions. Breast Cancer Res. 2003;5:31-36.

6. Wu T, Dai Y. Tumor microenvironment and therapeutic response. Cancer Letters, 2016:S0304383516300155.

7. Pitt J M , Marabelle A, Eggermont A, et al. Targeting the tumor microenvironment: removing obstruction to anticancer immune responses and immunotherapy. Annals of Oncology, 2016, 27(8):1482-1492.

8. Xiong $\mathrm{Y}$, Wang $\mathrm{K}$, Zhou $\mathrm{H}$ et al. Profiles of immune infiltration in colorectal cancer and their clinical significant: A gene expression-based study. Cancer Med. 2018 Sep;7(9):4496-4508.

9. Dave S S, Wright G, Tan B, et al. Prediction of Survival in Follicular Lymphoma Based on Molecular Features of Tumor-Infiltrating Immune Cells. NEJM, 2004, 351(21):2159.

10. Varun S N, Toor S M, Taha R Z, et al. DNA methylation and repressive histones in the promoters of PD-1, CTLA-4, TIM-3, LAG-3, TIGIT, PD-L1, and galectin-9 genes in human colorectal cancer. Clinical Epigenetics, 2018, 10(1):104-

11. Vougiouklakis T, Bao R, Nakamura Y, et al. Protein methyltransferases and demethylases dictate CD8+ T-cell exclusion in squamous cell carcinoma of the head and neck. Oncotarget, 2017, 8(68):112797-112808. 
12. Chevrier S, Levine JH, Zanotelli VRT, et al. An immune atlas of clear cell renal cell carcinoma. 2017;169(4):736-749 e18.

13. Zhang S, Zhang E, Long J et al. Immune infiltration in renal cell carcinoma. Cancer Sci. 2019 Mar 12.

14. Newman AM, Liu CL, Green MR, et al. Robust enumeration of cell subsets from tissue expression profiles. Nat Methods. 2015;12(5):453-457.

15. Charoentong $P$, Finotello $F$, Angelova $M$, et al. Pan-cancer immunogenomic analyses reveal genotypeimmunophenotype relationships and predictors of response to checkpoint blockade. Cell Rep. 2017;18(1):248-262.

16. Goodman, A. M. et al. Tumor Mutational Burden as an Independent Predictor of Response to Immunotherapy in Diverse Cancers. Mol Cancer Ter, 2017,16, 2598-2608.

17. Birkbak, N. J. et al. Tumor mutation burden forecasts outcome in ovarian cancer with BRCA1 or BRCA2 mutations. PLoS One. 2013, 8, e80023.

18. Klebanov N, Artomov M, Goggins WB et al. Burden of unique and low prevalence somatic mutations correlates with cancer survival. Sci Rep. 2019 Mar 19;9(1):4848.

19. Chalmers ZR, Connelly CF, Fabrizio D et al. Analysis of 100,000 human cancer genomes reveals the landscape of tumor mutational burden. Genome Med. 2017 Apr 19;9(1):34.

20. Wagner GP, Kin K, Lynch VJ. Measurement of mRNA abundance using RNA-seq data: RPKM measure is inconsistent among samples. Theory Biosci, 2012;131:281-5.

21. Ritchie ME, Phipson B, Wu D, Hu Y, Law CW, Shi W, et al. limma powers differential expression analyses for RNA-sequencing and microarray studies. Nucleic Acids Res 2015;43:e47-e.

22. Benjamini $Y$, Hochberg Y. Controlling the false discovery rate: a practical and powerful approach to multiple testing. J Royal Stat Soc Series B 1995;57:289-300.

23. Smyth GK. Linear models and empirical bayes methods for assessing differential expression in microarray experiments. Stat App/ Genet Mol Biol 2004;3:Article3.

24. Hibino N, Yi T, Duncan DR, Rathore A, Dean E, Naito Y, et al. A critical role for macrophages in neovessel formation and the development of stenosis in tissue-engineered vascular grafts. FASEB J.2011;25(12):4253-63.

25. Alvarez MM, Liu JC, Trujillo-de Santiago G, Cha BH, Vishwakarma A, Ghaemmaghami AM, and Khademhosseini A. Delivery strategies to control inflammatory response: modulating M1-M2 polarization in tissue engineering applications. J Control Release. 2015.

26. Martinez FO, Gordon S. The M1 and M2 paradigm of macrophage activation: time for reassessment. F1000Prime Rep. 2014;6:13.

27. Liu Y, Chen W, Minze L J, et al. Dissonant response of M0/M2 and M1 bone-marrow-derived macrophages to RhoA pathway interference. Cell and Tissue Research, 2016, 366(3):707-720.

28. Mami-Chouaib F, Blanc C, Corgnac S, et al. Resident memory T cells, critical components in tumor immunology. J Immunother Cancer. 2018;6(1):87. 
29. Rosenberg J, Huang J. CD8(+) T Cells and NK Cells: parallel and complementary soldiers of immunotherapy. Curr Opin Chem Eng. 2018;19:9-20.

30. Sprent J, Surh C D. Generation and maintenance of memory T cells. Current Opinion in Immunology, 2001, 21(2):167-172.

31. Zhao F, Zheng L, Goncearenco A, et al. Computational Approaches to Prioritize Cancer Driver Missense Mutations. International Journal of Molecular Sciences, 2018, 19(7).

32. Chapman, P.B. Hauschild, A. Robert, C. et al. Improved Survival with Vemurafenib in Melanoma with BRAF V600E Mutation. Engl. J. Med. 2011, 364, 2507-2516.

33. Karapetis, C.S. Khambata-Ford, S. et al. K-ras mutations and benefit from cetuximab in advanced colorectal cancer. Engl. J. Med. 2008, 359, 1757-1765.

34. Peters, U. Jiao, S. Schumacher, F.R. et al. Identification of genetic susceptibility loci for colorectal tumors in a genome-wide meta-analysis. Gastroenterology 2013, 144, 799-807.

35. Garcia-Closas, M. Chanock, S. Genetic susceptibility loci for breast cancer by estrogen receptor status. Clin. Cancer Res. 2008, 14, 8000-8009.

36. Eeles, R.A. Olama, A.A. Benlloch, S. et al. Identification of 23 new prostate cancer susceptibility loci using the icogs custom genotyping array. Nat. Genet. 2013, 45, 385-391.

37. Wimmer E, Ihrler S, Gires $\mathrm{O}$, et al. Fibroblast growth factor receptor 4 single nucleotide polymorphism Gly388Arg in head and neck carcinomas. World J Clin Oncol. 2019 Mar 24;10(3):136-148.

38. Li B, Huang Q, Wei GH. The Role of HOX Transcription Factors in Cancer Predisposition and Progression. Cancers (Basel). 2019 Apr 12;11(4). pii: E528.

39. Kubesova B, Pavlova S, Malcikova J, et al. Low-burden TP53 mutations in chronic phase of myeloproliferative neoplasms: Association with age, hydroxyurea administration, disease type and JAK2 mutational status. Leukemia, 2017.

40. Mukhopadhyay UK, Oturkar CC, Adams C, et al. TP53 Status as a Determinant of Pro- versus Antitumorigenic Effects of Estrogen Receptor-beta in Breast Cancer. J Natl Cancer Inst. 2019 Apr 16. pii: djz051.

41. Piotr M K, Agnieszka M K, Bogus Aw S, et al. Key Roles of Glutamine Pathways in Reprogramming the Cancer Metabolism[J]. Oxidative Medicine and Cellular Longevity, 2015, 2015:1-14.

\section{Table}

\section{TABLE 1. Primary tumor characteristics of HNSCC in TCGA.}

\section{Figures}




\begin{tabular}{|c|c|c|}
\hline Variable & Number of samples & $\%$ \\
\hline \multicolumn{3}{|l|}{ Gender } \\
\hline Male & 97 & 82.9 \\
\hline Female & 20 & 17.1 \\
\hline \multicolumn{3}{|c|}{ Age at diagnosis } \\
\hline$\leq 65$ & 77 & 65.8 \\
\hline$>65$ & 40 & 34.2 \\
\hline \multicolumn{3}{|l|}{ T-Stage } \\
\hline T1 & 7 & 6.0 \\
\hline T2 & 14 & 12.0 \\
\hline Т3 & 26 & 22.2 \\
\hline T4 & 55 & 47.0 \\
\hline Missing & 15 & 12.8 \\
\hline \multicolumn{3}{|l|}{ Grade } \\
\hline G1 & 8 & 6.8 \\
\hline G2 & 72 & 61.5 \\
\hline G3 & 32 & 27.4 \\
\hline G4 & 1 & 0.8 \\
\hline Missing & 4 & 3.4 \\
\hline \multicolumn{3}{|l|}{ Stage } \\
\hline I & 2 & 17.1 \\
\hline II & 10 & 8.5 \\
\hline III & 14 & 3.4 \\
\hline IV & 74 & 63.2 \\
\hline Missing & 17 & 14.5 \\
\hline \multicolumn{3}{|l|}{ M-stage } \\
\hline MO & 41 & 35.0 \\
\hline M1 & 1 & 0.8 \\
\hline Missing & 75 & 64.1 \\
\hline
\end{tabular}




\begin{tabular}{|lcc|}
\hline N-stage & & \\
N0 1 & 41 & 35.0 \\
N2 & 12 & 10.3 \\
N3 & 41 & 35.0 \\
Missing & 2 & 1.7 \\
\hline HNSCC, head and neck squamous cell carcinoma. \\
\hline
\end{tabular}
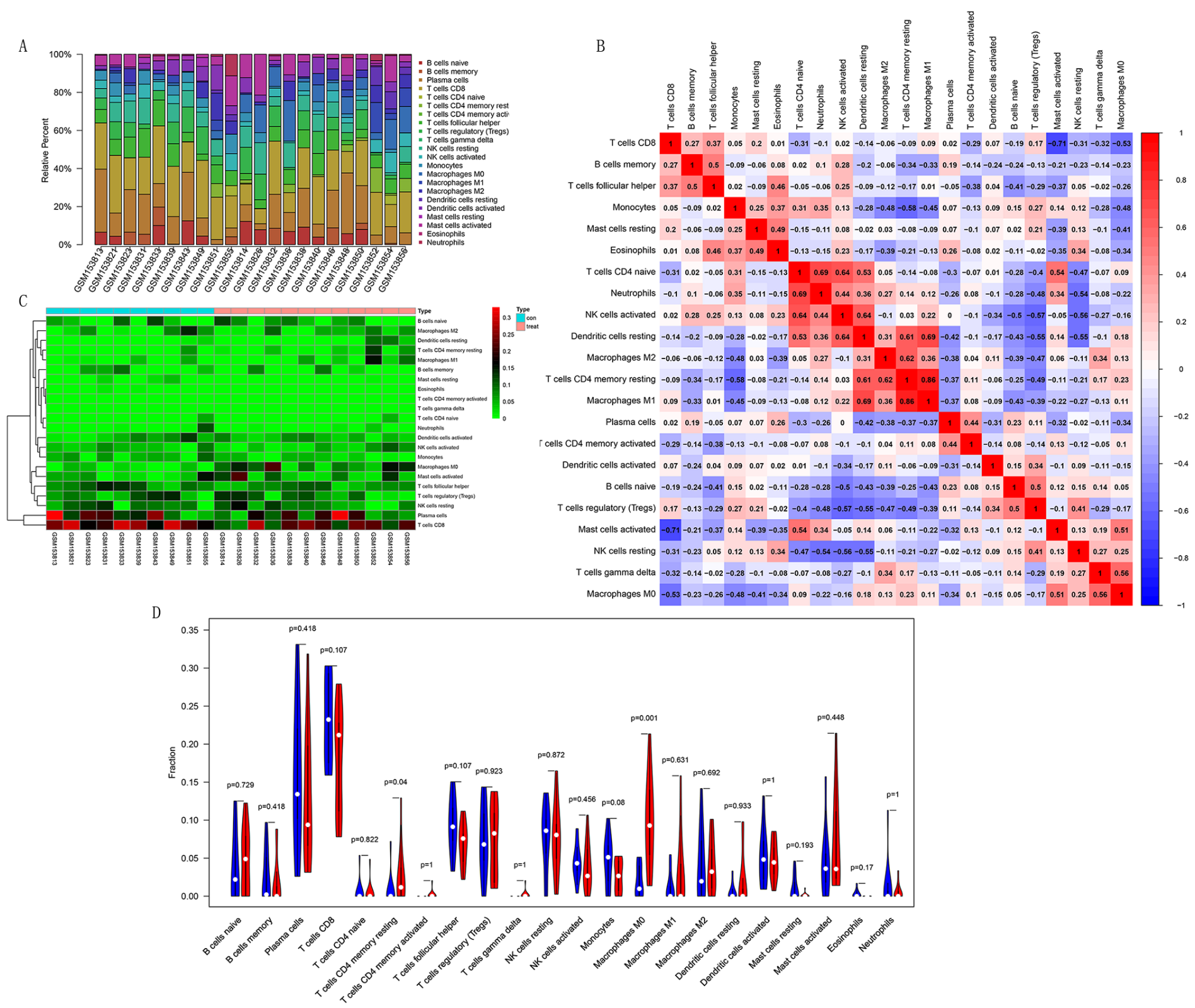

Figure 1

Boxplot, heatmap, corHeatmap and vioplot showed 22 TIICs subsets of immune response based on GEO (odd number is normal, even number is the tumor). The results suggested that the expression of 
macrophages $\mathrm{M} 0$ and $\mathrm{T}$ cells CD4 memory resting was significant difference and may plays an important role in regulate cancer progression $(P<0.05)$.
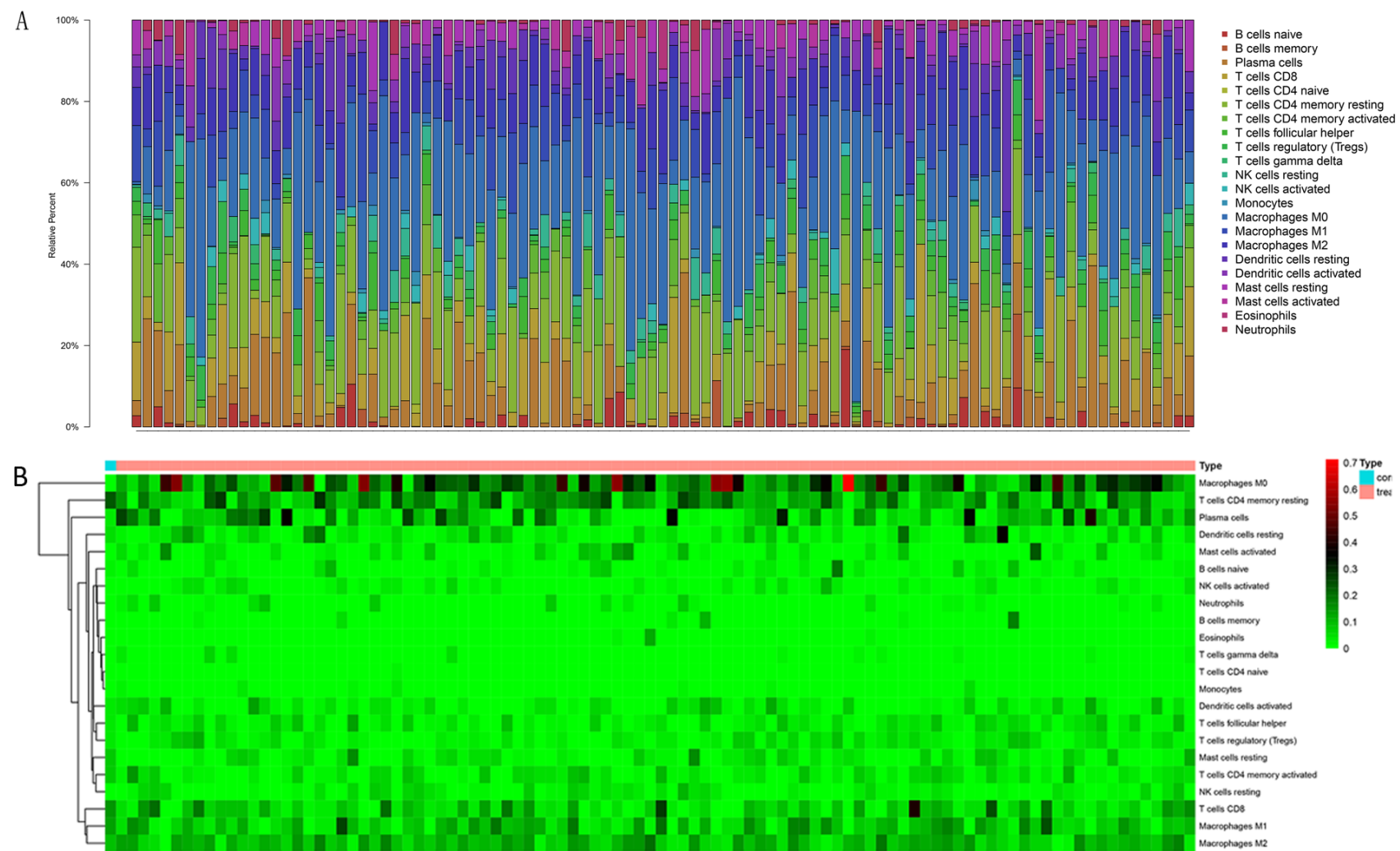

C

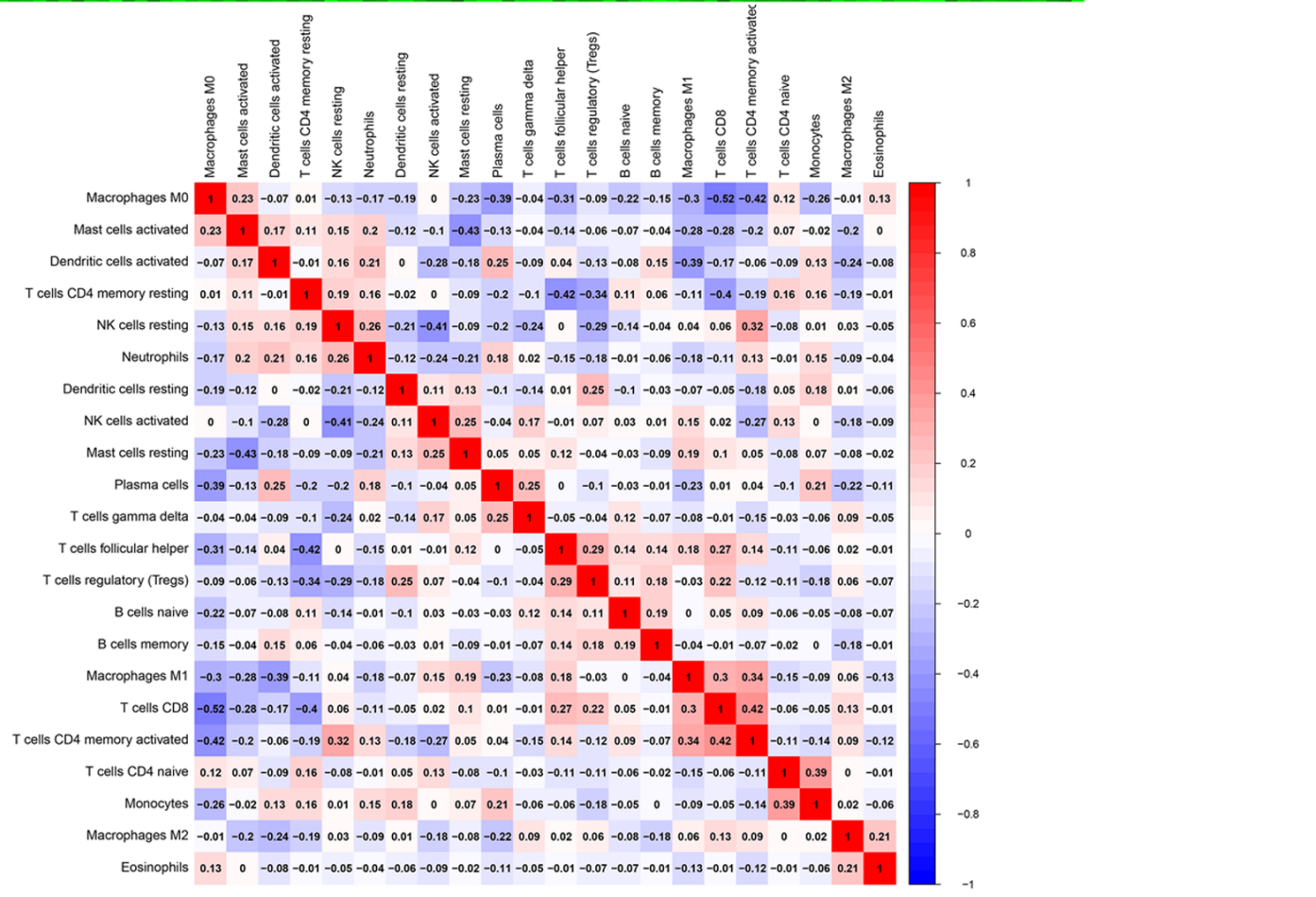

Figure 2

Boxplot, heatmap and corHeatmap visualization showed 22 TIICs subsets of immune response based on TCGA. 
A
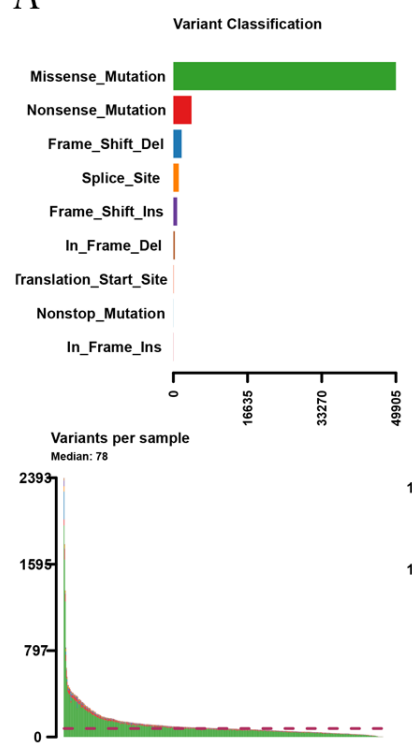

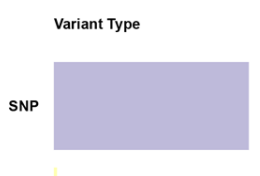

INS

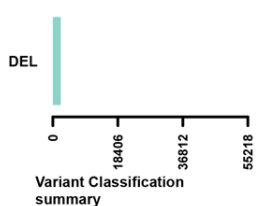

Variant $\mathrm{Cl}$
summary

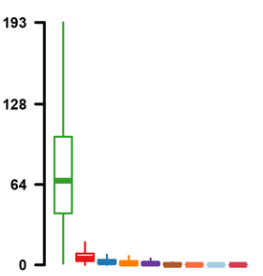

B
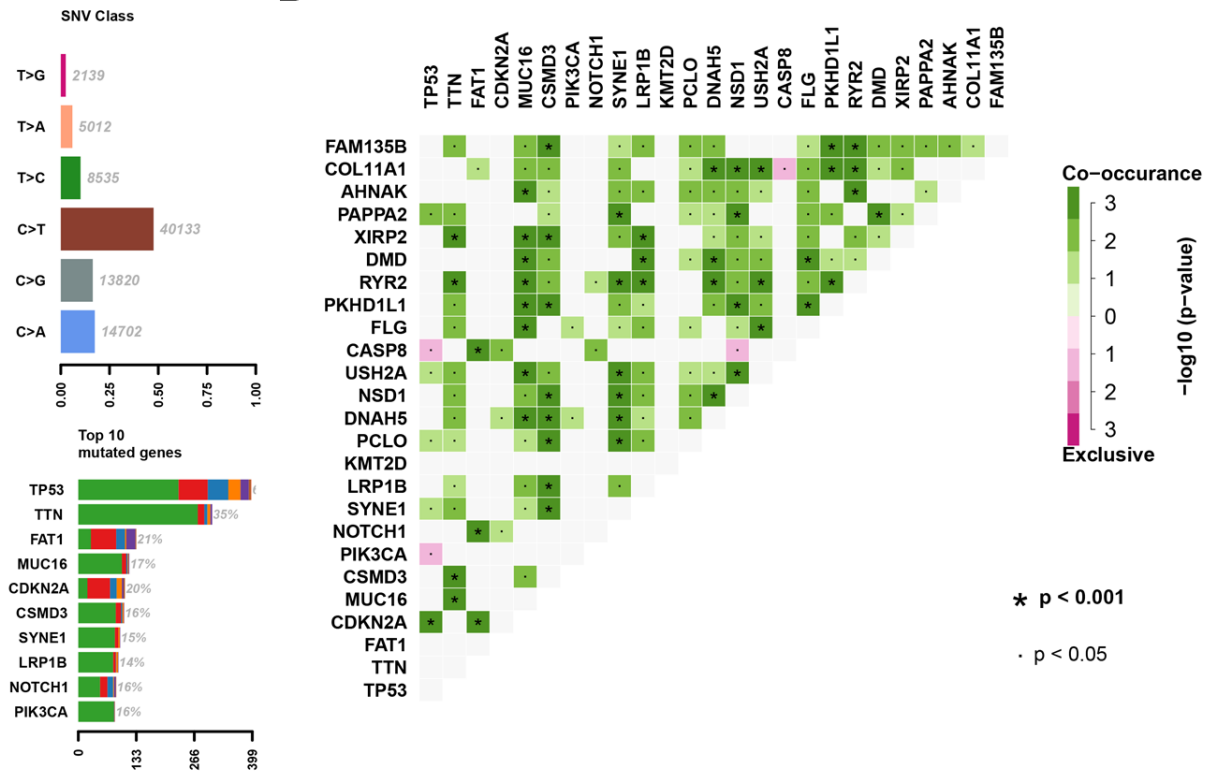

C

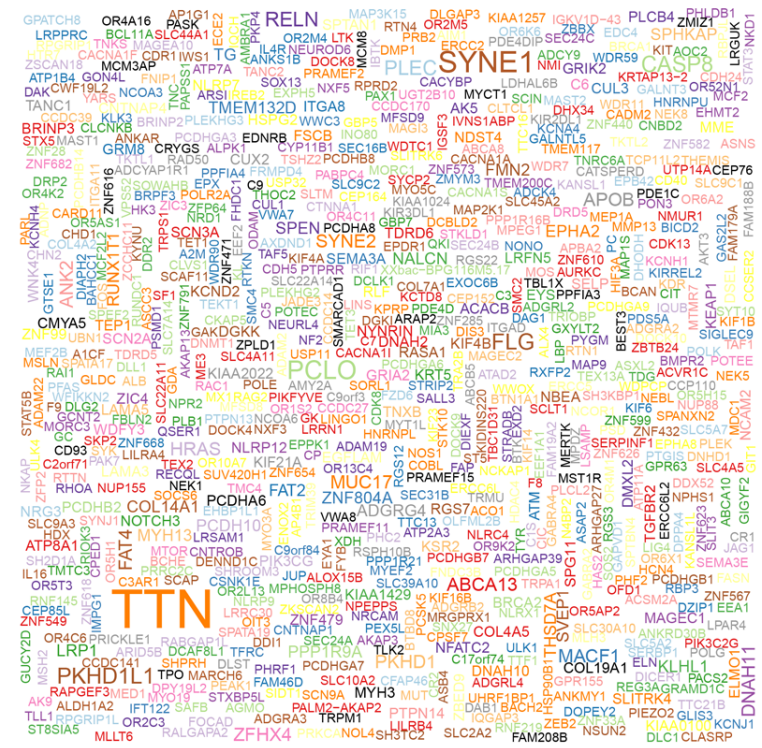

D

Altered in $478(94.47 \%)$ of 506 samples.

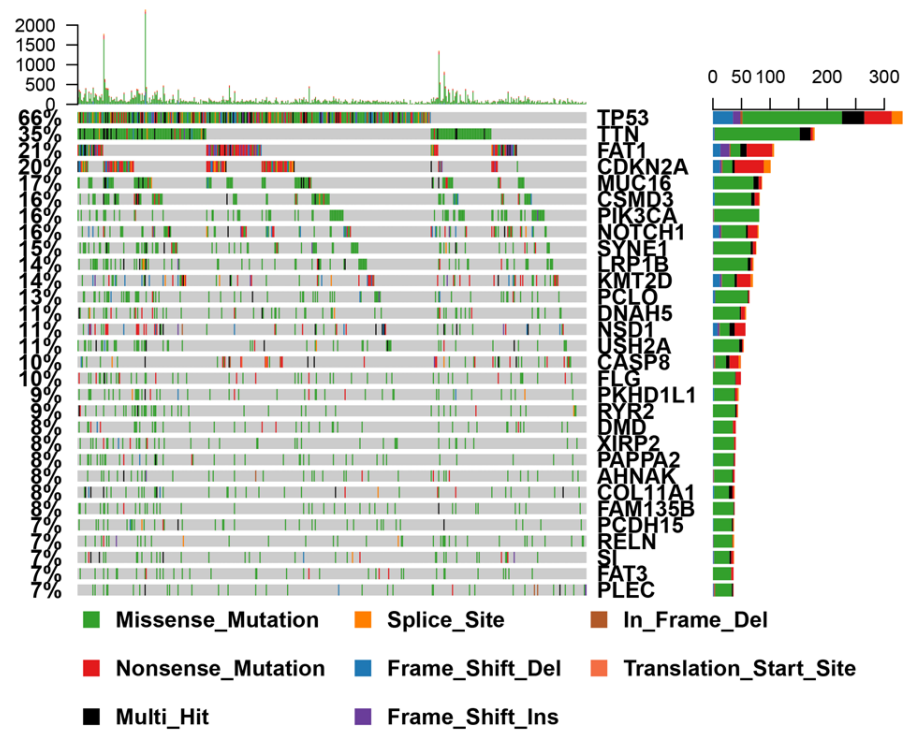

\section{Figure 3}

The result of tumor mutational burden in Head and Neck Cancer (cBioPortal). (A) The most common somatic mutations variant classification was missense mutation, the most common DNA sequence polymorphism type was SNP, the most common single nucleotide variants (SNV) class was $C>T$, the variants per sample median was 78 in HNSCC patients. Top 10 mutated genes that related to TMB was TP53, TTN, FAT1, MUC16, CDKN2A, CSMD3, SYNE1, LRP1B, NOTCH1 and PIK3CA. (B) CorHeatmap showed interaction between different mutated genes and the results showed that there are co-expression relationships among many mutated genes. (C-D) The gene cloudmap demonstrated that the most remarkable mutated genes were TTN and the waterfall plot tell us that the gene altered in $478(94.47 \%)$ of 506 samples as well as the relative percent, variant classification of every single mutated genes. 
A

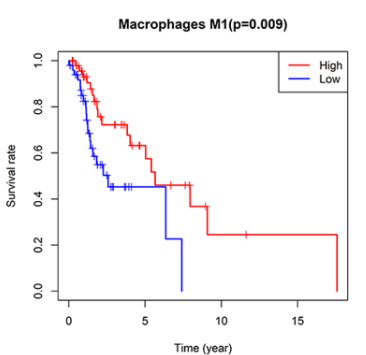

D

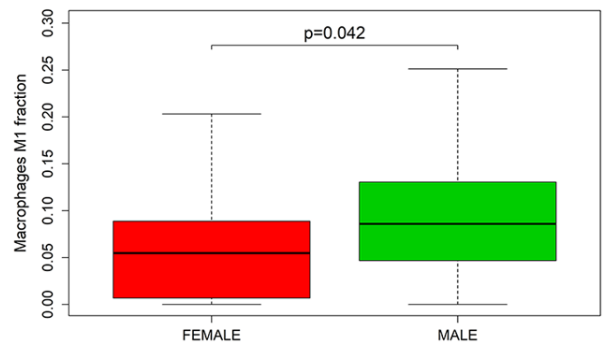

G
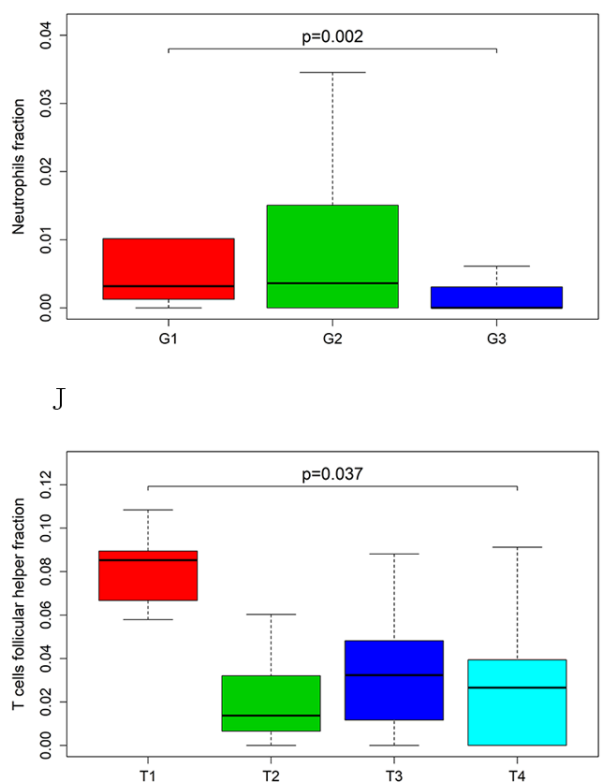

B

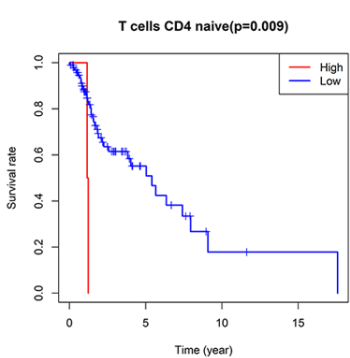

E

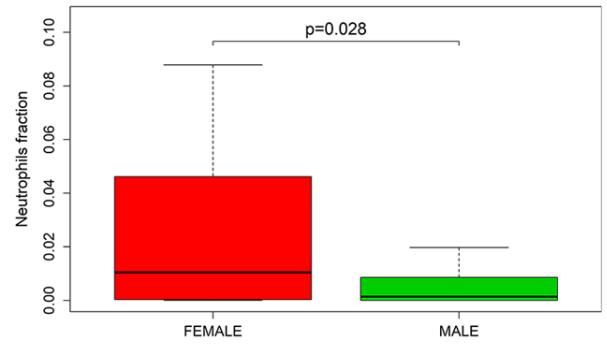

H

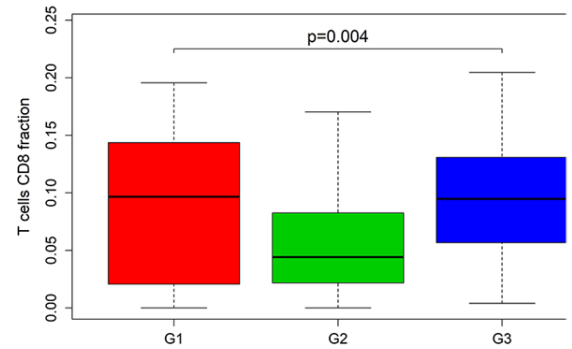

K

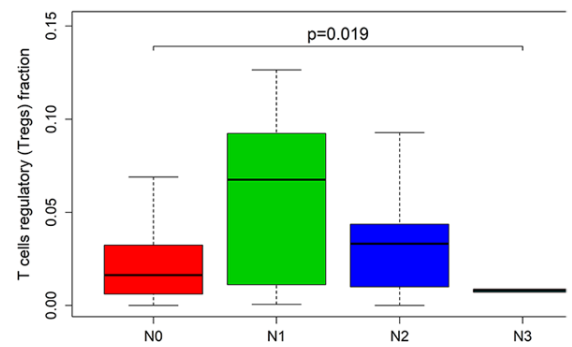

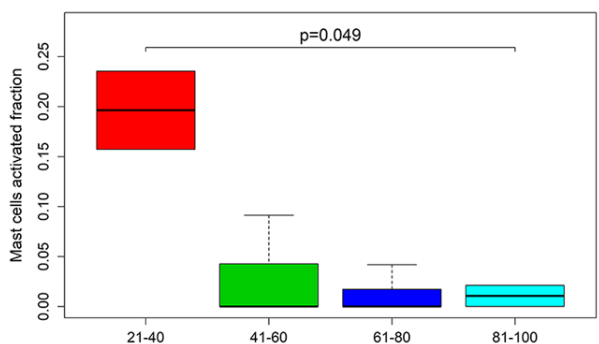

F

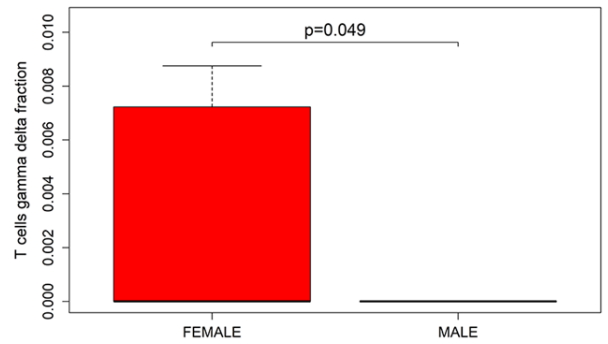

I

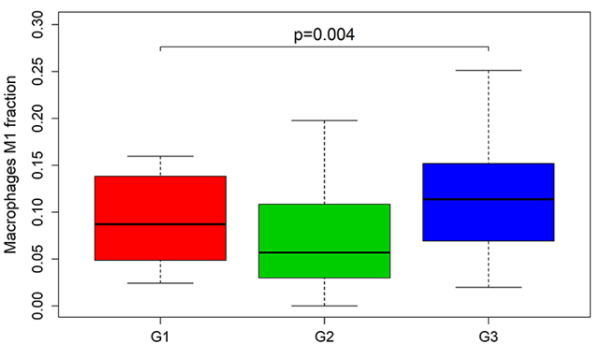

L

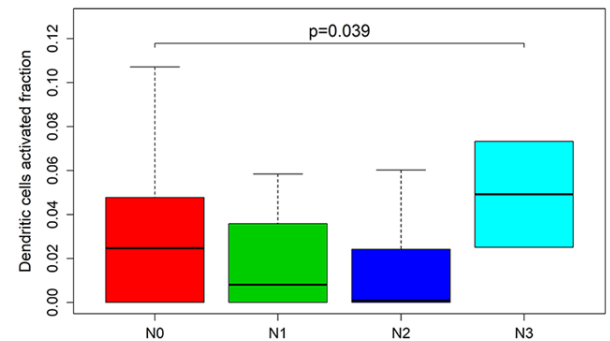

Figure 4

Survival rate between TMB and the relationship between tumor-infiltrating immune cells and patients' clinical factors. (A-B) The survival rate between TMB and macrophages M1 $(P=0.009)$ and T cells CD4 naïve. (C-L) The relationship between tumor-infiltrating immune cells and patients' clinical factors. 

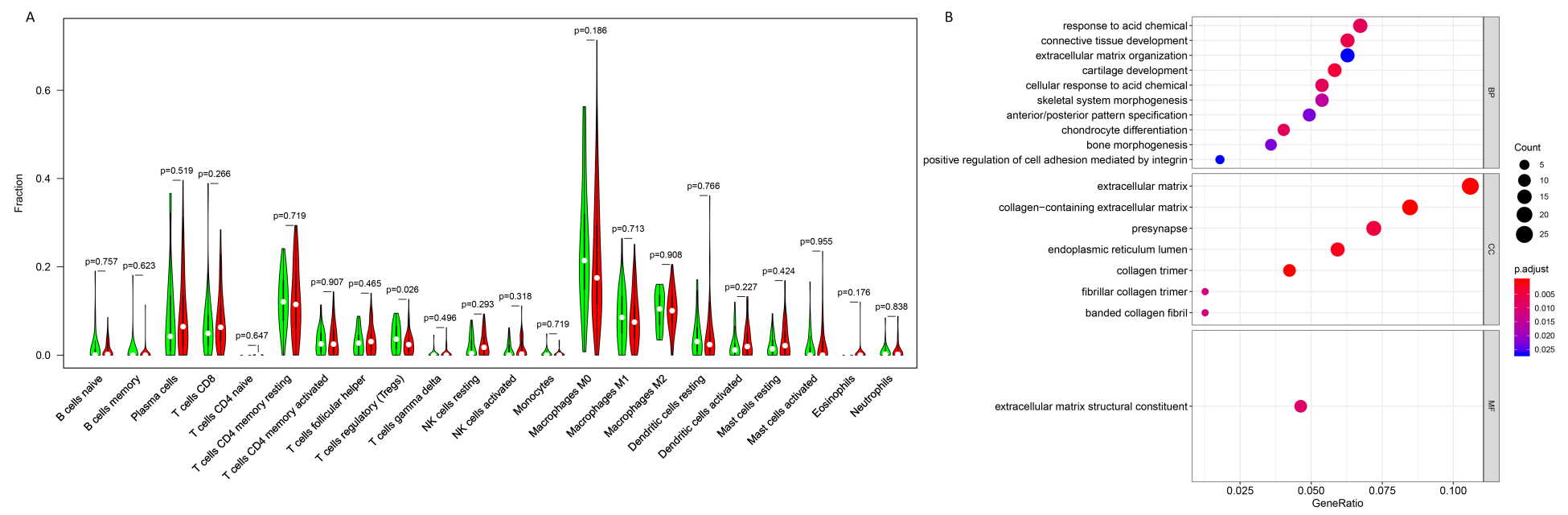

Figure 5

TMB and differentially expressed genes. (A) The clustering results. (B) GO analysis results. (C) The relationship between TMB and patients' gender. (D) The expression of T cells regulatory (Tregs) was significant difference and may plays an important role in regulate tumor cells $(P=0.026)$. (E) The relationship between TMB and patients' grade $(P=0.064)$. 